\title{
Obstructive sleep apnoea and cardiovascular comorbidity-growing evidence of independent association but recent doubts about benefits from therapy
}

Obstructive sleep apnoea (OSA) is highly prevalent, affecting up to $50 \%$ of the adult male general population (1) and is strongly associated with co-morbidity, especially cardiovascular (2). While the evidence that many co-morbidities such as cardiovascular, metabolic, hepatic, and malignancy are more prevalent in OSA, the evidence of independent association is less clear (3). Nonetheless, there is growing evidence that OSA is an independent risk factor for several co-morbidities, especially cardiovascular, and this evidence is strongest for more severe OSA (1). Cardiovascular disorders recognised to be more common in OSA include systemic hypertension, cardiac arrhythmias (especially atrial fibrillation), coronary artery disease, congestive heart failure, and stroke (2). Non-dipping nocturnal blood pressure is especially common in OSA (4) and carries a worse prognosis. The typical pathophysiological features of OSA such as intermittent hypoxia, fluctuating intrathoracic pressure, and recurring micro-arousals from sleep trigger a range of cell and molecular consequences that include sympathetic excitation, systemic inflammation and oxidative stress, in addition to metabolic and endothelial dysfunction (2), and various interactions of these intermediate mechanisms are proposed to contribute to the development of cardiovascular disease. Different mechanisms may predominate in individual co-morbidities. Sympathetic excitation appears to be particularly important in the development of hypertension (5) whereas systemic inflammation and oxidative stress may be more important in the development of atherosclerosis and coronary artery disease (6).

There is longstanding evidence of benefit to cardiovascular morbidity and mortality from effective therapy with nasal continuous positive airway pressure (CPAP) $(7,8)$. Short-term placebo-controlled studies have demonstrated lowering of blood pressure levels $(9,10)$, especially in more severe hypertension (11), but the magnitude of benefit is typically small. Previous long-term studies of cardiovascular outcomes have been observational in nature $(7,8)$, which carries a recognised risk of bias towards positive outcomes. In recent years, long-term randomised controlled trials of cardiovascular outcomes have cast doubt on potential benefits from CPAP therapy $(12,13)$. The recent SAVE trial report $(12)$ found no evidence of benefit from CPAP therapy in the secondary prevention of cardiovascular morbidity and mortality in patients with apnoea/hypopnea index (AHI) frequency per hour greater than 15 who were non-sleepy (Epworth Sleepiness Score less than 10). This multi-centre study involved 2,717 patients with pre-existing cardiovascular or cerebrovascular disease who were randomised to best usual care with or without CPAP therapy and followed for a mean period of 3.7 years. However, compliance with CPAP was relatively with an average 3.3 hours per night. Another randomized controlled long-term outcome study from a single centre (13) published around the same time as the SAVE report also found a similar lack of benefit to cardiovascular outcomes from CPAP therapy in patients with established cardiovascular disease, although there was evidence of benefit in more CPAP compliant patients. While both studies represent important contributions to the evidence base on this topic, both reports involved highly selected patient populations, and do not exclude potential benefits from CPAP therapy in the more typical OSA patients who are commonly sleepy, and in patients who comply with therapy (14).

The growing evidence of independent association of OSA with cardiovascular disease and the recent negative reports of benefit from CPAP outcome studies justify a detailed exploration of the association of OSA with cardiovascular diseases and their outcomes, which provides the rationale for this special supplement in the Journal. The collection of papers covers the most important cardiovascular disorders associated with OSA and are written by internationally recognised experts on the respective topics.

\section{Acknowledgements}

None. 


\section{References}

1. Heinzer R, Vat S, Marques-Vidal P, et al. Prevalence of sleep-disordered breathing in the general population: the HypnoLaus study. Lancet Respir Med 2015;3:310-8.

2. McNicholas WT, Bonsignore MR. Sleep apnoea as an independent risk factor for cardiovascular disease: current evidence, basic mechanisms and research priorities. Eur Respir J 2007;29:156-78.

3. Randerath W, Bassetti CL, Bonsignore MR, et al. Challenges and Perspectives in Obstructive Sleep Apnoea: Report by an ad hoc working group of the Sleep Disordered Breathing Group of the European Respiratory Society and the European Sleep Research Society. Eur Respir J 2018;52.

4. Seif F, Patel SR, Walia HK, et al. Obstructive sleep apnea and diurnal nondipping hemodynamic indices in patients at increased cardiovascular risk. J Hypertens 2014;32:267-75.

5. Crinion SJ, Ryan S, McNicholas WT. Obstructive sleep apnoea as a cause of nocturnal nondipping blood pressure: recent evidence regarding clinical importance and underlying mechanisms. Eur Respir J 2017;49.

6. Ryan S, Taylor CT, McNicholas WT. Systemic inflammation: a key factor in the pathogenesis of cardiovascular complications in obstructive sleep apnoea syndrome? Thorax 2009;64:631-6.

7. Marin JM, Carrizo SJ, Vicente E, et al. Long-term cardiovascular outcomes in men with obstructive sleep apnoea-hypopnoea with or without treatment with continuous positive airway pressure: an observational study. Lancet 2005;365:1046-53.

8. Doherty LS, Kiely JL, Swan V, et al. Long-term Effects of Nasal Continuous Positive Airway Pressure Therapy on Cardiovascular Outcomes in Sleep Apnea Syndrome. Chest 2005;127:2076-84.

9. Pepperell JC, Ramdassingh-Dow S, Crosthwaite N, et al. Ambulatory blood pressure after therapeutic and subtherapeutic nasal continuous positive airway pressure for obstructive sleep apnoea: a randomised parallel trial. Lancet 2002;359:204-10.

10. Durán-Cantolla J, Aizpuru F, Montserrat JM, et al. Continuous positive airway pressure as treatment for systemic hypertension in people with obstructive sleep apnoea: randomised controlled trial. BMJ 2010;341:c5991.

11. Becker HF, Jerrentrup A, Ploch T, et al. Effect of Nasal Continuous Positive Airway Pressure Treatment on Blood Pressure in Patients With Obstructive Sleep Apnea. Circulation 2003;107:68-73.

12. McEvoy RD, Antic NA, Heeley E, et al. CPAP for Prevention of Cardiovascular Events in Obstructive Sleep Apnea. N Engl J Med 2016;375:919-31.

13. Peker Y, Glantz H, Eulenburg C, et al. Effect of Positive Airway Pressure on Cardiovascular Outcomes in Coronary Artery Disease Patients with Nonsleepy Obstructive Sleep Apnea. The RICCADSA Randomized Controlled Trial. Am J Respir Crit Care Med 2016;194:613-20.

14. McNicholas WT. Continuous positive airway pressure therapy and cardiovascular outcomes in obstructive sleep apnoea syndrome: where are we now? J Thorac Dis 2016;8:E1644-6.

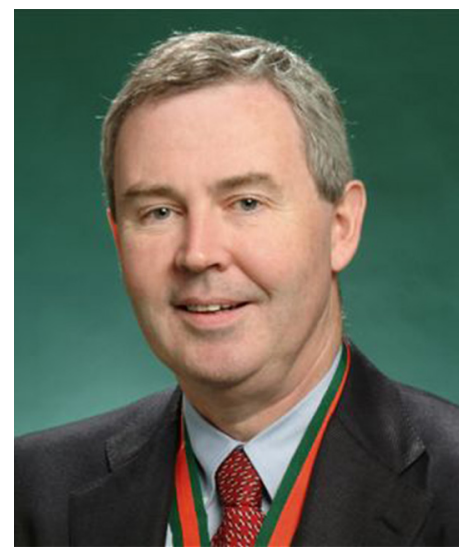

Walter T. McNicholas 


\section{Walter T. McNicholas ${ }^{1,2}$ \\ ${ }^{1}$ First Affiliated Hospital of Guangzhou Medical University, Guangzhou 510000, China; ${ }^{2}$ School of Medicine, University College Dublin, Dublin, Ireland. \\ (Email: walter:mcnicholas@ucd.ie) doi: $10.21037 /$ jtd.2018.10.118 \\ Conflicts of Interest: The author has no conflicts of interest to declare. View this article at: http://dx.doi.org/10.21037/jtd.2018.10.118}

Cite this article as: McNicholas WT. Obstructive sleep apnoea and cardiovascular comorbidity—growing evidence of independent association but recent doubts about benefits from therapy. J Thorac Dis 2018;10(Suppl 34):S4186-S4188. doi: 10.21037/jtd.2018.10.118 